\title{
Effects of Electroacupuncture on Alleviating Prostatodynia and Inflammation in Rats with Chronic Nonbacterial Prostatitis
}

\section{Chao Zhang \\ Ding $\mathrm{Li}$}

School of Acupuncture-Moxibustion and Tuina, Shanghai University of Traditional Chinese Medicine, Shanghai, People's Republic of China
Correspondence: Ding Li

Tel + 86-02I-5I322I78

Email liding020@yeah.net
Purpose: Chronic prostatitis (CP) is a common urinary disease characterized by a complex sequence of symptoms including prostatodynia, which results in significant physical pain and mental burden to affected patients. Currently, CP has no standard treatment. Thus, physical therapy electroacupuncture (EA) which has been successful in treating several inflammationrelated pain conditions was used to determine its effect on rats with $\mathrm{CP}$.

Materials and Methods: Rats were castrated and treated with beta-estradiol for 28 days for CP modeling, and EA was initiated one week after. Following three weeks of treatment, painrelated behavioral testing, mechanical withdrawal threshold, and potential histopathological mechanisms were examined.

Results: EA treatment produced a significant antinociceptive effect, effectively increasing the pain threshold of CP rats, and the biochemical results revealed significantly lowered prostatic specific antigen levels. Histopathological results also demonstrated that EA exerted protective properties on prostate morphological changes, as well as decreased inflammation cytokines and oxidative stress molecular expressions in prostate tissue. Furthermore, EA alleviated microglial and astrocyte activation in the dorsal horn of the spinal cord, decreasing CXC motif ligand 1 expressions in activated spinal astrocytes.

Conclusion: In conclusion, it was demonstrated that EA alleviated CP-induced pain, which was partly achieved by the downregulation of inflammation, oxidative stress, and spinal cord glial activation.

Keywords: chronic prostatitis, prostatodynia, inflammation, spinal cord, glial activation

\section{Introduction}

Chronic prostatitis (CP) is one of the most frequently occurring andrological and urological diseases, accounting for more than $90 \%$ of prostatitis outpatients. ${ }^{1,2}$ Generally, CP affects middle-aged men as a group of clinical symptoms called chronic prostatitis/chronic pelvic pain syndrome (CP/CPPS), which has been reported to have serious outcomes. ${ }^{3,4}$ In addition to urogenital symptoms, such as frequent urination, urgency, pain, nocturia, erectile dysfunction, premature ejaculation, and painful ejaculation, $\mathrm{CP} / \mathrm{CPPS}$ is often accompanied by other pain related symptoms, including perineal pain and lower abdominal discomfort, ${ }^{5,6}$ which greatly impact the quality of life.

Accumulating evidence has shown that hormones play an important role in the susceptibility of inflammation and autoimmune response during the occurrence of prostatitis. ${ }^{7,8}$ Particularly, the production of proinflammatory cytokines under the action of initiating factors, including infection, endocrine disorders, autoimmunity, and trauma, 
increases the expressions of cyclooxygenase-2 (COX-2), inducible nitric oxide synthase (iNOS), prostaglandin (PSA), and intercellular adhesion factor, among others. ${ }^{9,10}$ In turn, these increased factors damage the prostate tissue, consequently resulting in the many symptoms of prostatitis through their respective pathways. For example, long term noxious inflammatory stimulation often affects the nervous system, resulting in numerous neurological manifestations, with pain as one of the most common clinical complaints in $\mathrm{CP} .{ }^{11}$ Moreover, the heat and pain sensitivity of the vulva and pelvic floor have also been reported to change, indicating an increased sensitivity of the central nervous system in CP patients. ${ }^{12}$ In addition to these involvements of the nervous system, a previous study has shown that the immunoreactive region of substance $P$ was increased in the L6 to S2 spinal cord (sixth lumbar and second sacral spinal nerves, respectively) segments of $\mathrm{CP}$ rats, suggesting that the cause of $\mathrm{CP}$ pain was related to the secondary lesion of the spinal cord which innervated the prostate. ${ }^{13}$ Consistent with this, glial cells distributed in the central and peripheral nervous system, which are mainly composed of microglia and astrocytes, play a supportive and nutritional role, as well as participate in the central regulation of pain. As such, the continuous activation of glial cells in the spinal cord may possibly contribute to the characteristics of chronic persistent and refractory pain in $\mathrm{CP} .{ }^{14}$

Despite these findings, the original lesion in $\mathrm{CP}$ is not always consistent with the target location of pain symptoms. Moreover, existing therapeutic drugs cannot simultaneously act on the prostate and spinal cord for pain alleviation. Fortunately, physical therapy electroacupuncture (EA) has been shown to be effective in regulating inflammation and improving pain thresholds in animal models of neuropathic pain. ${ }^{15,16}$ Mounting evidence reveals that the Zusanli acupuncture point is an effective target for EA, which has been involved in regulating chronic neuropathic pain and its implicated inflammatory processes. $^{17-19}$ Thus, with the goal of developing a new strategy to intervene in $\mathrm{CP}$, the aim of this study was to observe the effect of EA on the inflammatory response and pain management in rats with chronic nonbacterial prostatitis.

\section{Materials and Methods}

\section{Animals}

A total of 30 male Sprague Dawley rats weighing $250 \pm$ $20 \mathrm{~g}$ were obtained from the Shanghai Laboratory Animal Management Centre. Animal studies were approved by the
Shanghai University of Traditional Chinese Medicine Institutional Animal Care and Use Committee, and all experiments were conducted in accordance with the National Institute of Health guidelines for animal care. The animals were housed in an specific-pathogen-free (SPF)-conditions facility with controlled ambient temperature $\left(24 \pm 1{ }^{\circ} \mathrm{C}\right)$ and humidity (50-60\%). Following this, 20 rats were castrated, given penicillin to avoid postoperative infection, and then subcutaneously injected with $17 \beta$-estradiol $(0.25 \mathrm{mg} / \mathrm{kg} / \mathrm{day}$, Sigma, USA, dissolved in corn oil) for four weeks. ${ }^{20}$ Specifically, the rats were randomly divided into three groups: (1) 10 castrated rats without EA intervention were classified as the model group, (2) the other 10 castrated rats with EA intervention were classified as the EA group, and (3) the remaining 10 rats with the sham operation were classified as the control group (Con group; injected with corn oil). Afterwards, the L6-S2 spinal cord were of all rats were collected for analysis.

\section{Electroacupuncture Therapy}

In a quiet and comfortable environment, the rats were placed in the self-made fixation device (shown in Supplementary 1) from 8:00 a.m. to 10:00 a.m. every day, which allows the exposure of their limbs and tail but restricts movement. After the rats settled down, bilateral Zusanli acupoints were selected and acupuncture needles (diameter: $0.25 \mathrm{~mm}$; length: $30 \mathrm{~mm}$; Huatuo, Suzhou Medical Supplies Factory Co., Ltd., Suzhou) were inserted with a depth of about $5 \mathrm{~mm}$, as indicated in a previous study. ${ }^{21}$ The electrical stimulator (Great Wall, KWD.808, Danton Medical Equipment Co., Ltd., Hangzhou) was then connected to the two acupuncture needles, and continuous waves (frequency: $2 \mathrm{~Hz}$, intensity: $3 \mathrm{MA}$ ) of electrical stimulation were given. This was performed once a day for 20 minutes each, from the 7 th day after modeling for a total of 21 days, as previously described. ${ }^{22,23}$

\section{Hematoxylin Eosin and Immunohistochemistry Staining}

After the rats were decapitated, the prostate tissue was separated and hematoxylin eosin (HE) sections were made. Inflammatory cell accumulation was then observed and scored. Following the instructions of the commercial Rat IL-1 $\beta$ Kit (ZK-R3160, Zike Bio, Shenzhen), paraffin sections of the prostate were processed, and 
immunohistochemistry was performed, wherein brown cytoplasmic granules indicated positive staining. Similarly, after the spinal cord was removed, paraffin sections were made. After blocking with 5\% BSA, anti-GFAP (1:200, SC-6170, Santa Cruz Biotechnology) and anti-Iba1 (1:300, ab5076, Abcam) were added intravenously at $4{ }^{\circ} \mathrm{C}$ overnight. The second IgG polyclonal antibody labeled with horseradish peroxidase was then incubated for 30 minutes at room temperature. After being washed with PBS for three times, DAB was dripped at room temperature for color development. Lignin was then dyed, differentiated, dehydrated, made transparent, and was finally sealed with neutral gum. The Image $J$ image analysis software was used to calculate the average stain intensity.

\section{Enzyme-Linked Immunosorbent Assay}

Blood and prostate tissues were collected for biochemical determinations including prostatic specific antigen (PSA), IL-1 $\beta$, IL-6, TNF- $\alpha$, malondialdehyde (MDA), COX-2, prostaglandin E2 (PEG2), and iNOS, using a commercial kit (Abcam), as previously described. ${ }^{24}$

\section{Paw Withdrawal Threshold}

Paw withdrawal threshold (PWT) was tested every seven days using the Semmes-Weinstein von Frey Touch Test Sensory Evaluator (North Coast Medical Inc.), as previously described. ${ }^{25,26}$ Specifically, rats were placed in a plexiglass cage with a metal mesh at the bottom. After 5 minutes of adaptation, they were stimulated by von Frey hairs to make the cilia bend until the rats showed the foot retraction reflex. When the rats had the behavior of lifting or licking their feet, the positive reaction time and number of grams ( $\mathrm{g}$ ) were recorded.

\section{Western Blot}

As previously described, ${ }^{27}$ after the spinal cord tissues were sonicated and centrifugated, the supernatant was collected and quantified using the BCA Protein Assay Kit (Thermo Fisher Scientific). Equal amounts of protein sample were then separated by $10 \%$ SDS-PAGE and transferred to PVDF membranes (PerkinElmer). Afterwards, blots were incubated in the appropriate primary antibody: GFAP (1:1000, SC-6170, Santa Cruz Biotechnology), Ibal (1:800, ab5076, Abcam), and GAPDH (1:1000, 2118, Cell Signaling Technology). After washing, the membranes were incubated with peroxidase-conjugated affinity secondary antibody. For analysis, protein levels were normalized to GAPDH on the same gel, and all quantifications were performed using the AlphaEase FC software (Alpha Innotech).

\section{Immunostaining}

After perfusion with $0.1 \mathrm{M}$ of PBS and 4\% PFA, the spinal cords were immersed in 30\% sucrose solution for dehydration. Spinal cords segments were then cut into $30 \mu \mathrm{m}$-thick sections and processed for 1 hour by blocking in 5\% BSA containing $0.3 \%$ Triton $\mathrm{X}-100$ at room temperature. Afterwards, the cut segments were incubated with mouse anti-GFAP (MAB360, 1:500, Millipore) and rabbit antiCXC motif ligand 1 (CXCL1) (BAO21312, 1:200; BOSTER) at $4{ }^{\circ} \mathrm{C}$ overnight. Subsequently, the segments were washed with PBS thrice and were incubated with the appropriate secondary antibodies (1:1000, Invitrogen) for 1 hour at room temperature. Images were then acquired using confocal microscopes (Zeiss), which were analyzed using the ImageJ and Photoshop (Adobe) software.

\section{Statistical Analyses}

The results were presented as means \pm SEM. Parametric statistical analysis was applied by one-way ANOVA using the SPSS 19.0 program (SPSS Inc, Chicago, IL, USA). Statistical significance for all analyses was set at $\mathrm{p}<0.05$.

\section{Results}

\section{EA Alleviates the Pain Response of $C P$ Rats}

PWT was monitored during EA intervention (at 0, 7, 14, 21, and 28 days) to evaluate the pain response in each group and assess the effect of EA on CP rats. On assessment, PWT was almost constant in the Con group, whereas the threshold of the $\mathrm{CP}$ group continuously decreased during the modeling period. Furthermore, EA treatment effectively rescued PWT in the EA group as compared to that in the model group (Figure 1).

\section{Effect of EA on Prostate Damage in CP Rats}

Following four weeks of intervention, we collected prostate and blood tissues to assess the effect of EA. The HE staining results of rat prostate tissues showed the following: (1) the prostate histological structure of the Con group was clear and the gland cavity was regular; (2) pathological changes in the prostate tissues of the $\mathrm{CP}$ group showed an irregular and disordered gland cavity, with a large number of infiltrated inflammatory cells in the stroma and dilated and congested blood vessels; and lastly, (3) the prostate histological 


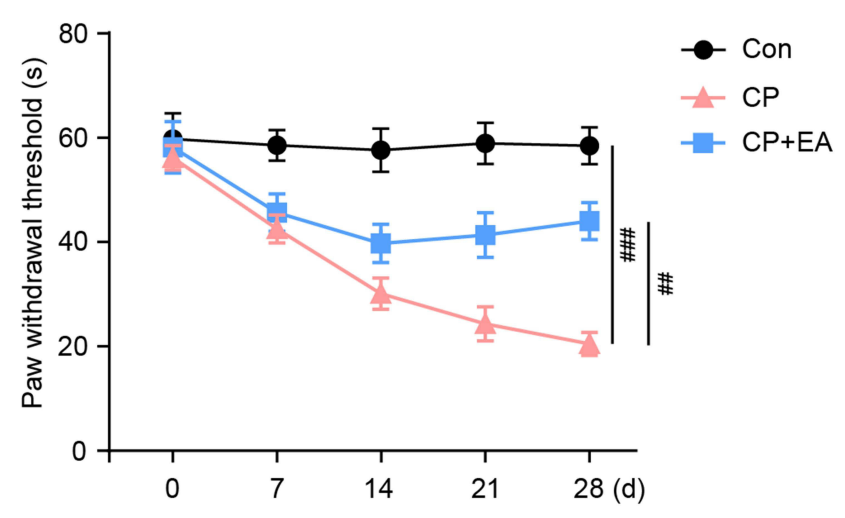

Figure I EA treatment attenuates the pain response of CP rats. Paw withdrawal threshold were assessed at each time point $(0,7,14,21,28$ day) during the $C P$ modeling and EA treatment for each group. Data are presented as mean $\pm \mathrm{SEM}, \mathrm{n}=$ 10 rat per group. Bonferroni One-way ANOVA were used for multiple comparisons, ${ }^{\#} \mathrm{p}<0.01,{ }_{\mathrm{P}}<0.001$.

structure of the EA group was significantly alleviated after EA administration (Figure 2A and B). Furthermore, serum PSA results showed that EA significantly reduced the increase of PSA levels induced by CP modeling (Figure 2C).

\section{Effect of EA on Prostate Inflammation in CP Rats}

Since inflammatory response is one of the pathological manifestations of $\mathrm{CP}$, we examined local cytokine expressions after EA treatment. On assessment of IL-1 $\beta$ immunohistochemistry (IHC) staining in prostate tissue, as shown in Figure $3 \mathrm{~A}$, the positive signal area was obviously reduced following EA treatment. Moreover, the concentrations of inflammatory cytokines (IL-1 $\beta$, IL-6, TNF- $\alpha$ ) in the model group were higher than those in the Con group, whereas concentrations of these cytokines were decreased in the EA group as compared to those in the model group (Figure 3B-D).

\section{Regulatory Effect of EA on Prostate Oxidative Stress in CP Rats}

Endogenous oxidative stress enzymatic regulating systems, such as MDA, COX-2, PEG2, and iNOS, were examined using enzyme-linked immunosorbent assay.

\section{A}

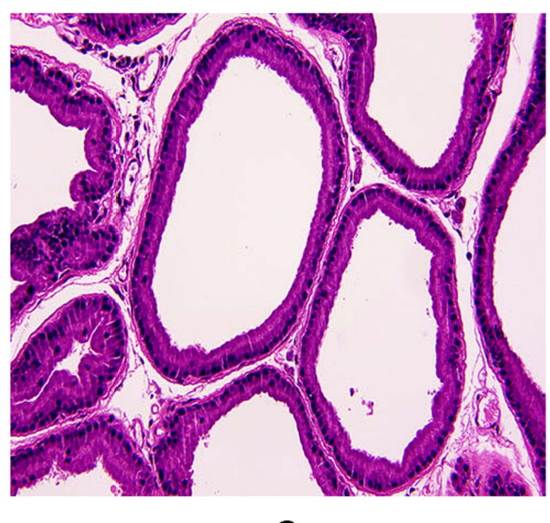

Con

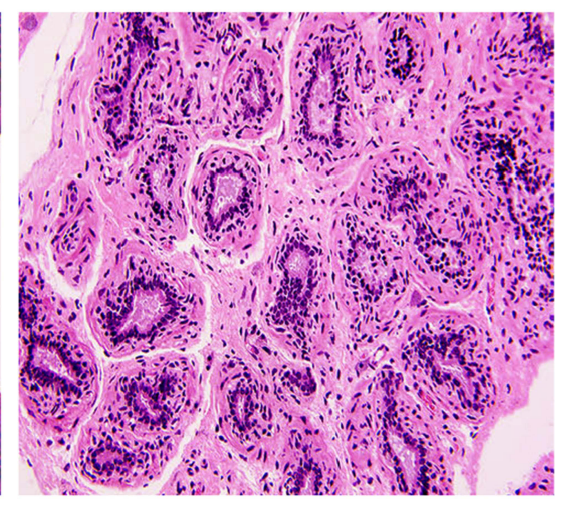

$\mathrm{CP}$

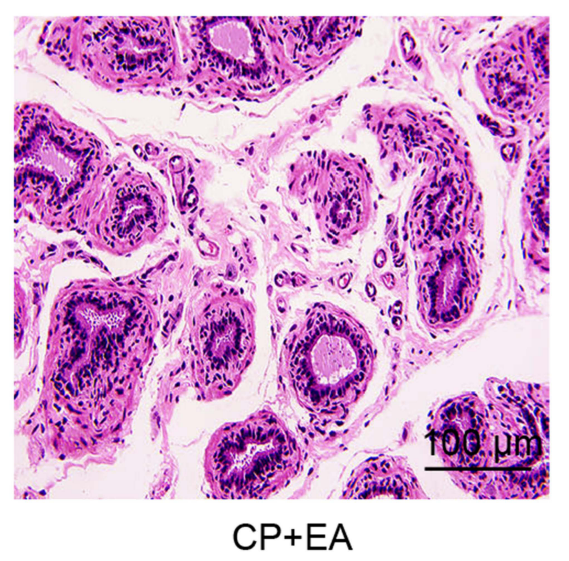

$\mathrm{CP}+\mathrm{EA}$
B

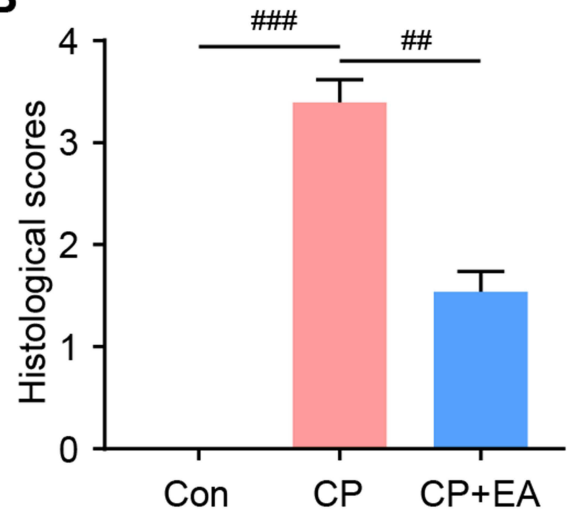

C

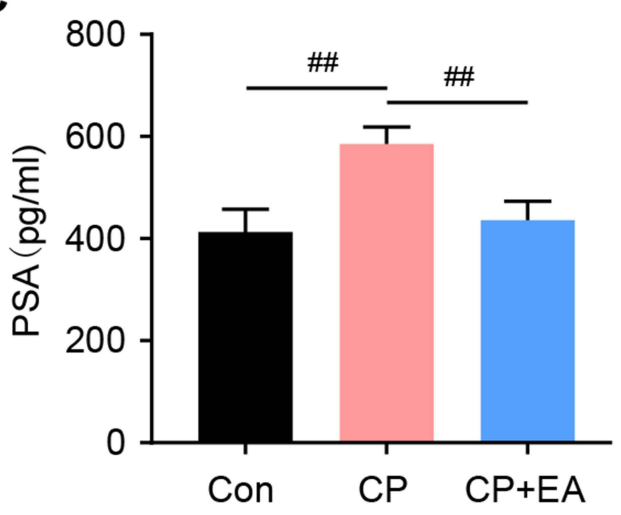

Figure 2 Electroacupuncture(EA) treatment attenuates prostate damage of CP rats. (A) The HE staining of prostate sections in each group. Scale bar= $100 \mu$ m. (B) The tissue histological score of the prostate damage. (C) Statistical results of the blood PSA level of each group. Data are presented as mean \pm SEM, $n=8$ rat per group. Bonferroni One-way ANOVA were used for multiple comparisons, ${ }^{\#} \mathrm{p}<0.01,{ }^{\#} \mathrm{p}<0.001$. 
A
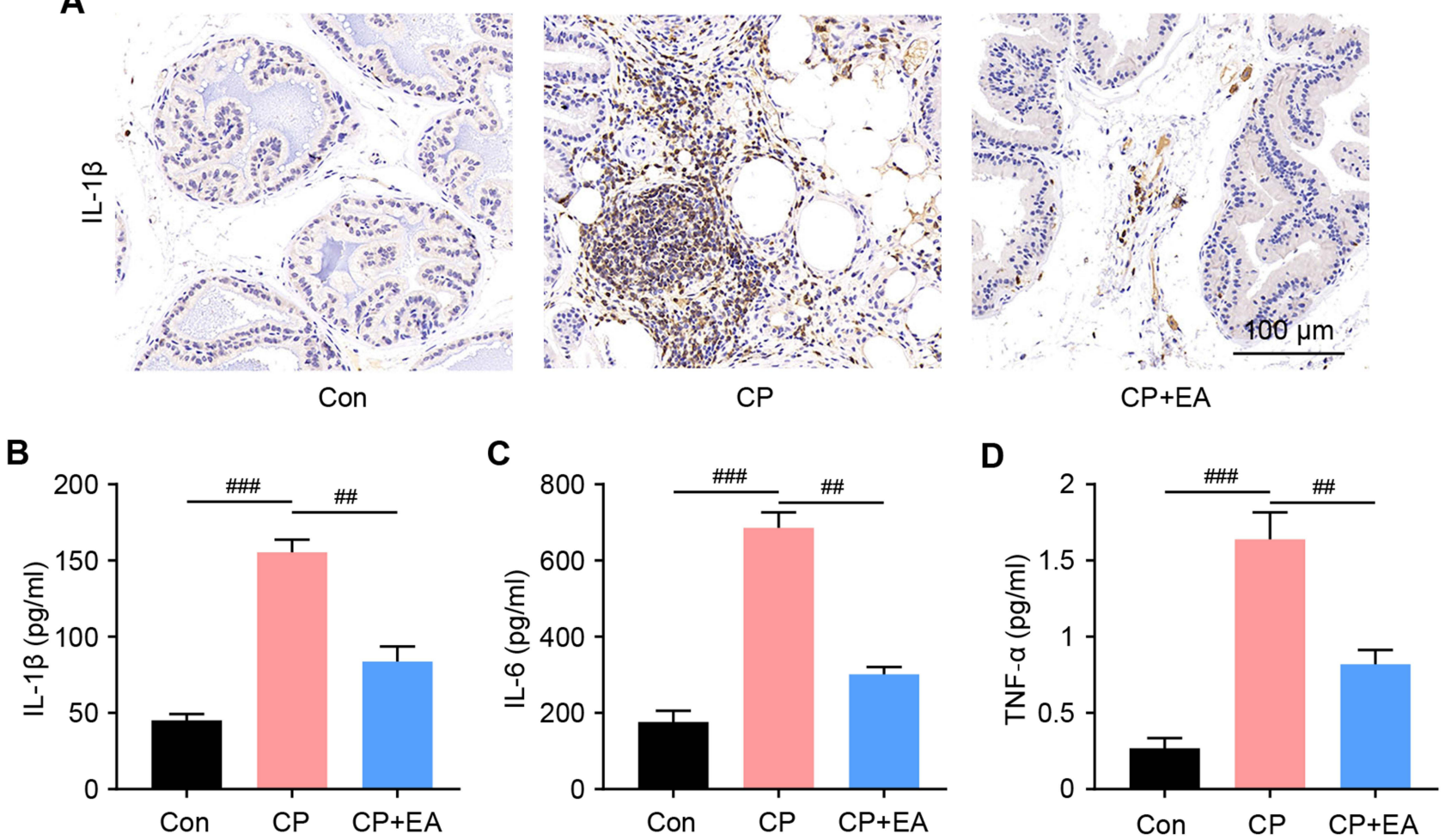

Figure 3 EA treatment decrease the inflammation response of CP rats. (A) The IHC staining of IL-I $\beta$ in prostate of each group. Scale bar=I00 $\mu$ m. (B-D) The tissue histological score of the prostate damage. Effect of EA treatment on IL-I $\beta$, IL-6, and TNF- $\alpha$ levels in the prostate of rats. Each assay was repeated for three times. Data are

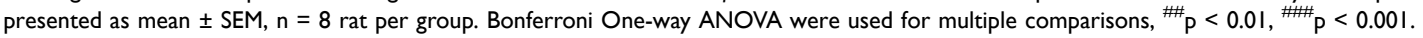

Results showed that expressions of MDA, COX-2, PEG2, and iNOS were higher in the model group than in the Con group. In contrast, their expressions in the EA group were significantly decreased as compared to those in the model group (Figure 4A-D).

\section{EA Inhibits Spinal Cord Glial Activation in CP Rats}

Accumulating evidence has shown that spinal cord glial activation contributes to $\mathrm{CP}$ pain. Thus, we examined the distribution and expression of astrocytes (GFAP) and microglia (Iba1) in the spinal cord of each group. On modeling, $\mathrm{CP}$ induction was found to activate the astrocyte and microglia, which was confirmed with the Western blot results (Figure 5A-D). Conversely, EA treatment significantly inhibited astrocyte and microglial activation, consequently decreasing GFAP and Ibal expressions. In addition, since CXCL1 is mainly expressed in astrocytes and is implicated in its activation of mediated pain sensitization, the immunoreactivity of CXCL1 and GFAP of the spinal cord was also examined. Results showed that while most astrocytes expressed CXCL1 in CP rats, decreased CXCL1 expressions in the astrocytes were observed in the EA group (Figure 5E).

\section{Discussion}

In this study, the animal model for chronic nonbacterial prostatitis was established by castration and estrogen injection. After EA, the pathological changes, inflammation, and oxidative stress reaction of the prostate were significantly improved, and the pain symptoms were alleviated. Given these results, it can be said that EA inhibited microglial and astrocyte activation in the spinal cord. Regulation of CXCL1 expression in astrocytes may be the mechanism involved in the pain management of $\mathrm{CP}$ by EA. Additionally, based on findings from histology, morphology, the specific antigen, inflammatory factors, and pain threshold, it was shown that $17 \beta$-estradiol could induce $\mathrm{CP}$ syndrome in a statistically significant number of rats in both the model and EA groups. Thus, we believe that the study findings are convincing since the model was applicable in both groups.

Given its numerous symptoms, CP pathogenesis has been reported to be complex, in which hormonal changes are not the only responsible factors for the development of this condition. ${ }^{28-30}$ As a common rat $\mathrm{CP}$ model, the CP hormone model has shown that the histopathological morphology of rat prostatitis is consistent with the 
A

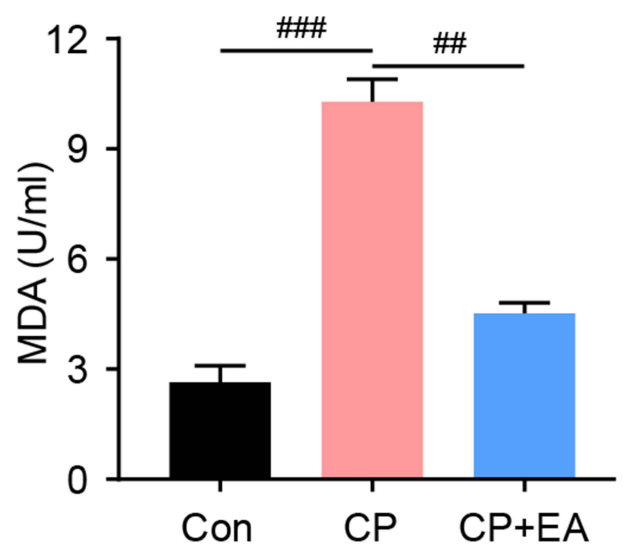

C

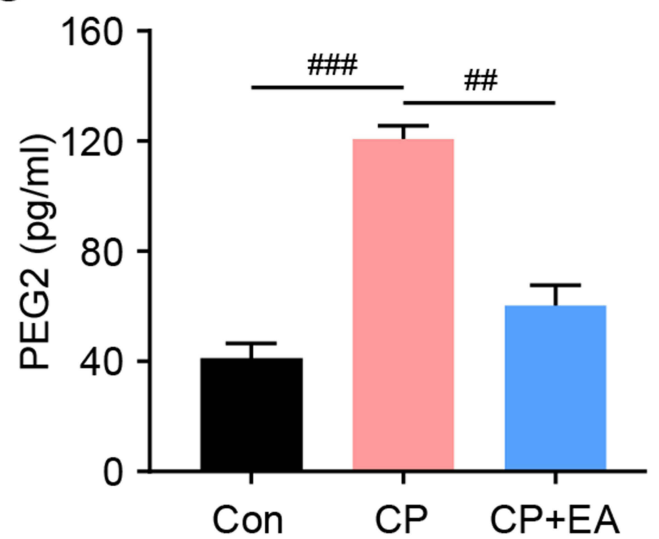

B

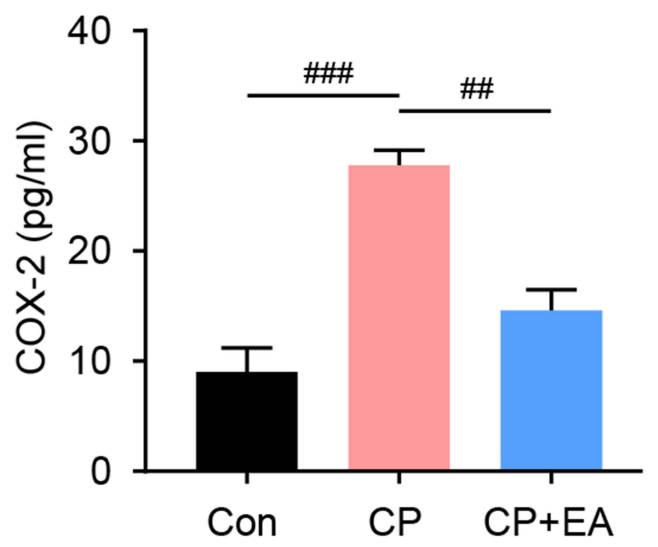

D

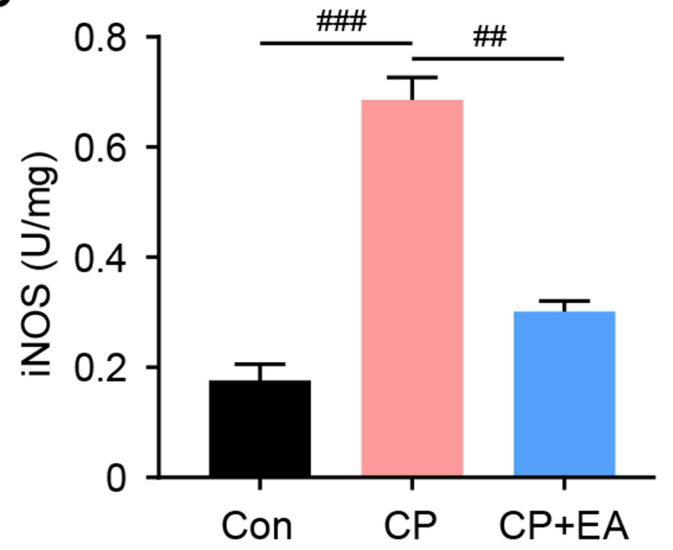

Figure 4 EA treatment decrease the oxidative stress of CP rats. Effect of EA on oxidative stress in CP rats: (A) MDA; (B) COX-2; (C) PEG2; (D) iNOS. ELISA assay was repeated for three times. Data are presented as mean $\pm \mathrm{SEM}, \mathrm{n}=8$ rat per group. Bonferroni One-way ANOVA were used for multiple comparisons, ${ }^{\#} \mathrm{P}<0.0 \mathrm{I},{ }^{\ldots \ldots} \mathrm{P}<0.00 \mathrm{I}$.

characteristics of human chronic nonbacterial prostatitis, ${ }^{28}$ providing an excellent tool for clinical studies researching about the pathological changes and reactions of CP. In this study, after four weeks of EA, we confirmed its therapeutic effect on CP through the morphological examination of prostate tissues. Moreover, serum PSA concentrations after prostate injury were reported to be significantly reduced after EA treatment, and thus we detected the related inflammatory factors in prostate tissues to further determine the efficacy of EA. Results showed that the concentrations of IL-1 $\beta$, IL- 6 , and TNF- $\alpha$ in the prostate tissue of the model group were significantly increased, and similar to previous findings, concentrations measured in the EA group were reported to be significantly decreased, indicating the procedure's potential for cytokine reduction and local oxidative stress regulation. In addition to the classic inflammatory factors, PEG2 was also decreased significantly after EA. This is especially crucial as the role of PEG2 in inflammatory pain has been recognized, ${ }^{31}$ which is closely related to the pain symptoms of prostatitis. Therefore, the reduction of inflammatory factors and oxidative stress response can be said to alleviate local pathological changes, protect the prostate, and control pain responses.

In particular, prostatodynia is the most common symptom of $\mathrm{CP}$ and the main reason for patients to seek a doctor. ${ }^{32}$ Studies have shown that the activation of spinal cord glia are closely related to hyperalgesia and pain persistence, which is characteristic of prostatodynia. ${ }^{14,33}$ Once activated, astrocytes form a positive feedback with microglia, releasing neuroexcitatory substances that can drive pain sensitization. ${ }^{34}$ Moreover, since the spinal cord is wrapped in a myelin membrane, it is difficult for common drugs to penetrate the spinal cord, resulting in a prostatic pain that has been characterized as chronic, persistent, refractory, and migratory in pain localization. ${ }^{35}$ As established earlier, glial cells, including astrocytes and microglia, are closely related to the occurrence and development of prostatitis pain. ${ }^{14,36} \mathrm{In}$ 
A

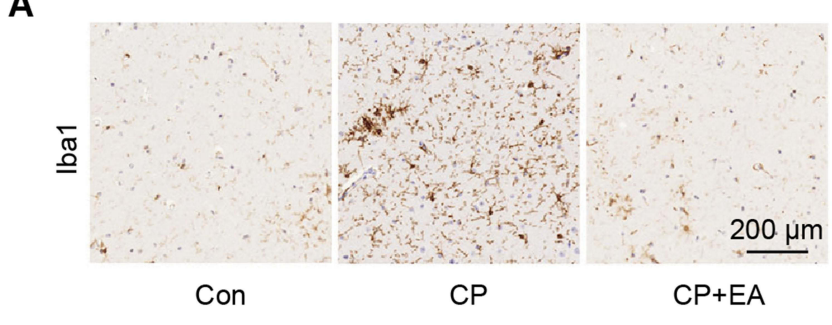

B
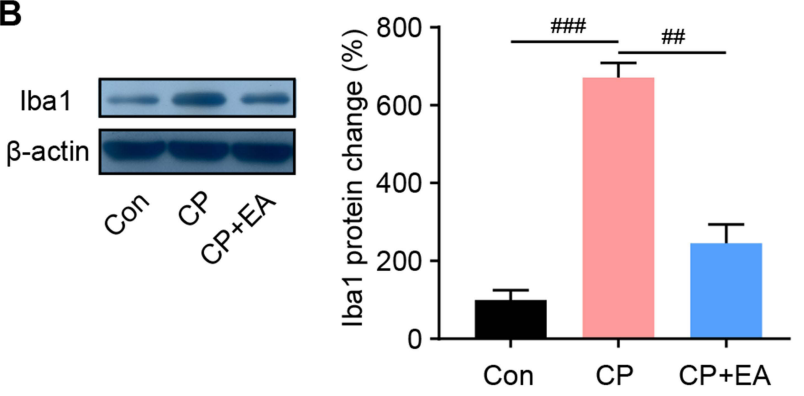

E
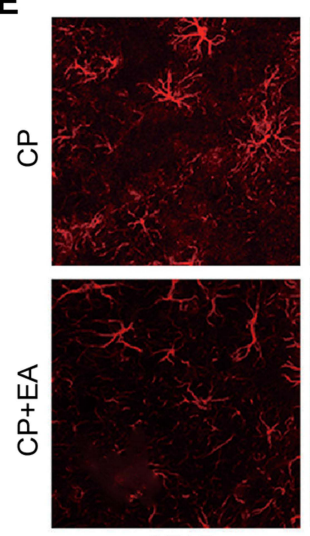

GFAP
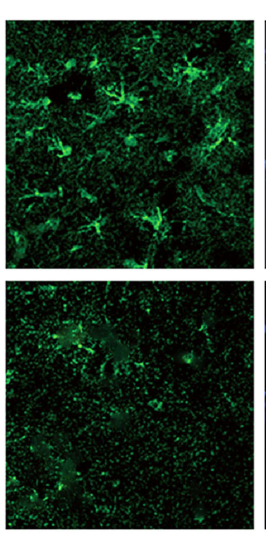

CXCL1
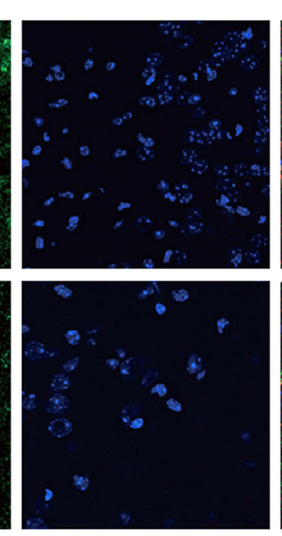

DAPI
C

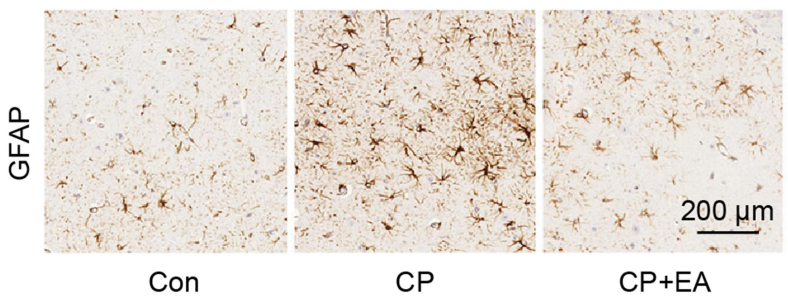

D
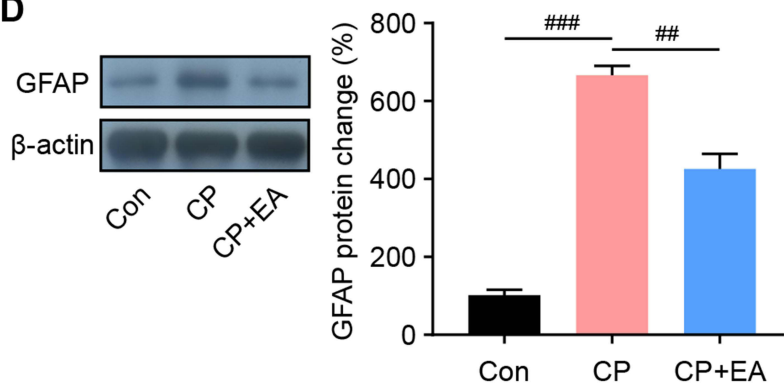

CXCL1/GFAP

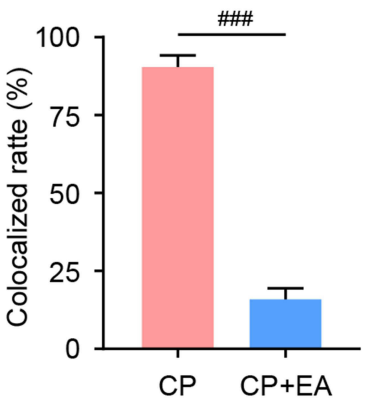

Figure 5 EA treatment inhibited the astrocyte and microglia activation in spinal cord of CP rats. (A) The IHC staining of microglia in spinal cord of each group. Scale bar=200 $\mu$ m. (B) The protein level of microglia market lbal in spinal cord of each group. (C) The IHC staining of astrocyte in spinal cord of each group. Scale bar=200 $\mu \mathrm{m}$. (D) The protein level of astrocyte market GFAP in spinal cord of each group. (E) Immunofluorescence shows expression of CXCLI (green) and GFAP (red) in rat spinal cord after EA treatment. Data are presented as mean $\pm \mathrm{SEM}, \mathrm{n}=8$ rat per group. Bonferroni One-way ANOVA were used for multiple comparisons, ${ }^{\prime \prime}<0.01$, ${ }^{m} \mathrm{p}<0.00 \mathrm{I}$.

that regard, studies have shown the ability of EA to regulate inflammatory substance release and glial cell activation in the spinal cord. ${ }^{37-39}$ In the present study, immunohistochemistry showed that GFAP and Ibal expressions in the L5-S2 segments were enhanced in the dorsal horn of the spinal cord among the model group rats. In contrast, EA treatment showed an inhibitory effect on the activation of two immune related cells in the spinal cord among the EA group rats. In addition to GFAP and Iba1, CXCL1 is another important inflammatory pathway in astrocytes, ${ }^{40}$ which is mainly expressed on astroglia and has been established to contribute to other conditions of neuralgia. ${ }^{26}$ In the present study, EA treated rats displayed decreased CXCL1 levels, suggesting that CXCL1 plays a wide role in spinal cord associated neuralgia in CP.
A previous study reported that the EA stimulation of the "Zusanli" acupoint could effectively inhibit the expressions of glial cell related pathways in the spinal cord, ${ }^{41}$ which is similar to the results of our study. Additionally, CXCL1 has been reported to be the astroglue in the spinal cord, which mediates neuralgia in bone cancer. ${ }^{26}$ As such, we speculated that astroglial CXCL1 in the spinal cord is a therapeutic target for EAmediated pain desensitization, and that the "Zusanli" acupoint, as one of the commonly recognized acupoints with analgesic effect, could be utilized with EA. However, it should be noted that we were lacking in other choices for acupoints and parameters for the comparison of the therapeutic effect of EA. In addition, only CXCL1 was selected to establish a limited preliminary link for the study, and no verification experiment was designed for the present study. Therefore, further studies 
focusing on the selection of acupoints and the optimization of parameters are effective strategies to improve these findings.

In conclusion, EA can significantly reduce inflammatory cell infiltration in $\mathrm{CP}$, inhibit inflammatory reaction and oxidative stress, and play a protective therapeutic role. Furthermore, EA can improve hyperalgesia, inhibit spinal cord glial cell activation, and reduce CXCL1 expression, which is the notable mechanism of EA in the treatment of CP.

\section{Data Sharing Statement}

The datasets used or analysed during the current study are available from the corresponding author on reasonable request.

\section{Consent for Publication}

All authors agreed for the publication of this paper.

\section{Funding}

This work was supported by Xinglin Inheritance Talent Training Program of Shanghai University of Traditional Chinese Medicine.

\section{Disclosure}

The authors declare that they have no competing interests.

\section{References}

1. Wazir J, Ullah R, Li S, et al. Efficacy of acupuncture in the treatment of chronic prostatitis-chronic pelvic pain syndrome: a review of the literature. Int Urol Nephrol. 2019;51(12):2093-2106. doi:10.1007/ s11255-019-02267-2

2. Xiong S, Liu X, Deng W, et al. Pharmacological interventions for bacterial prostatitis. Front Pharmacol. 2020;11:504. doi:10.3389/ fphar.2020.00504

3. Zhang J, Zhang X, Cai Z, Li N, Li H. The lifetime risk and prognosis of chronic prostatitis/chronic pelvic pain syndrome in the middle-aged Chinese males. Am J Mens Health. 2019;13(4):1557988319865380. doi: $10.1177 / 1557988319865380$

4. Zhang Y, Ma H, Nan T, et al. Comparative efficacy of oral Chinese patent medicine for chronic prostatitis/chronic pelvic pain syndrome with sexual dysfunction: a Bayesian network meta-analysis of randomized controlled trials. Front Pharmacol. 2021;12:649470. doi:10.3389/fphar.2021.649470

5. Lee M, Seo D, Lee C, et al. Relationship between hypogonadal symptoms, sexual dysfunction and chronic prostatitis in middle-aged men by self-reported questionnaires, even without biochemical testosterone deficiency. World J Mens Health. 2020;38(2):243-249. doi:10.5534/wjmh.190117

6. Nickel J, Freedland S, Castro-Santamaria R, Moreira D. Chronic prostate inflammation predicts symptom progression in patients with chronic prostatitis/chronic pelvic pain. J Urol. 2017;198(1):122-128. doi:10.1016/j.juro.2017.01.035

7. Kurita M, Yamaguchi H, Okamoto K, Kotera T, Oka M. Chronic pelvic pain and prostate inflammation in rat experimental autoimmune prostatitis: effect of a single treatment with phosphodiesterase 5 inhibitors on chronic pelvic pain. Prostate. 2018;78(15):1157-1165. doi: $10.1002 /$ pros. 23690
8. Lotti F, Rastrelli G, Maseroli E, et al. Impact of metabolically healthy obesity in patients with andrological problems. J Sex Med. 2019;16 (6):821-832. doi:10.1016/j.jsxm.2019.03.006

9. Diserio GP, Nowotny E. Experimental autoimmune prostatitis: in vivo induction of the autoimmune response to lymphocytic soluble factors. alterations at the endocrine metabolism level. Am J Reprod Immunol. 2011;39(4):226-234. doi:10.1111/j.1600-0897.1998. tb00358.x

10. Pontari M, Ruggieri M. Mechanisms in prostatitis/chronic pelvic pain syndrome. $J$ Urol. 2004;172(3):839-845. doi:10.1097/01.ju.00 00136002.76898 .04

11. Tripp D, Curtis nickel J, Landis J, Wang Y, Knauss J. Predictors of quality of life and pain in chronic prostatitis/chronic pelvic pain syndrome: findings from the National Institutes of Health Chronic Prostatitis Cohort Study. BJU Int. 2004;94(9):1279-1282. doi:10.1111/j.1464-410X.2004.05157.x

12. Saini R, Fitzgerald JP, Gonzalez RR, Gulmi FA. Urodynamic findings in chronic prostatitis and chronic pelvic floor pain. Curr Prostate Rep. 2008;6(4):177-181. doi:10.1007/s11918-008-0026-y

13. Ishigooka M, Nakada T, Hashimoto T, Iijima Y, Yaguchi H. Spinal substance $\mathrm{P}$ immunoreactivity is enhanced by acute chemical stimulation of the rat prostate. Urology. 2002;59(1):139-144. doi:10.1016/ s0090-4295(01)01470-4

14. Deng G, Lu M, Zhao Y, Yuan Y, Chen G. Activated spinal astrocytes contribute to the later phase of carrageenan-induced prostatitis pain. $J$ Neuroinflammation. 2019;16(1):189. doi:10.1186/s12974-0191584-3

15. Wang L, An J, Song S, et al. Electroacupuncture preserves intestinal barrier integrity through modulating the gut microbiota in DSS-induced chronic colitis. Life Sci. 2020;261:118473. doi:10.1016/j.1fs.2020.118473

16. Yen C, Hsieh C, Lin Y. Electroacupuncture reduces chronic fibromyalgia pain through attenuation of transient receptor potential vanilloid 1 signaling pathway in mouse brains. Iran J Basic Med Sci. 2020;23(7):894-900. doi:10.22038/ijbms.2020.39708.9408

17. Xu Z, Fang J, Xiang X, et al. Electroacupuncture alleviates pain-related emotion by upregulating the expression of NPS and its receptor NPSR in the anterior cingulate cortex and hypothalamus. eCAM. 2020;2020:8630368. doi:10.1155/2020/8630368

18. Zhang R, Zhu B, Wang L, et al. Electroacupuncture alleviates inflammatory pain via adenosine suppression and its mediated substance $P$ expression. Arq Neuropsiquiatr. 2020;78(10):617-623. doi:10.1590/ 0004-282x20200078

19. Liu Q, Liu Y, Bian J, Li Q, Zhang Y. The preemptive analgesia of pre-electroacupuncture in rats with formalin-induced acute inflammatory pain. Mol Pain. 2019;15:1744806919866529. doi:10.1177/ 1744806919866529

20. Lu J, Su Y, Chen X, et al. Rapamycin-induced autophagy attenuates hormone-imbalance-induced chronic non-bacterial prostatitis in rats via the inhibition of NLRP3 inflammasome-mediated inflammation. Mol Med Rep. 2019;19(1):221-230. doi:10.3892/mmr.2018.9683

21. Huang C, Li H, Shi Y, Han J, Wan Y. Ketamine potentiates the effect of electroacupuncture on mechanical allodynia in a rat model of neuropathic pain. Neurosci Lett. 2004;368(3):327-331. doi:10.1016/ j.neulet.2004.07.073

22. Lee D, Jiu Y, Hsieh C. Electroacupuncture at Zusanli and at Neiguan characterized point specificity in the brain by metabolomic analysis. Sci Rep. 2020;10(1):10717. doi:10.1038/s41598-020-67766-0

23. Song M, Okuno S, Orito K, Chen W, Kamiie J. Electroacupuncture improves neuronal function by stimulation of ascending peripheral nerve conduction in rats with spinal cord injury. J Trad Chin Med. 2019;39(4):509-515.

24. Zong Y, Chen T, Dong H, Zhu L, Ju W. Si-Ni-San prevents reserpine-induced depression by inhibiting inflammation and regulating CYP450 enzymatic activity. Front Pharmacol. 2019;10:1518. doi:10.3389/fphar.2019.01518 
25. Lv S, Zhang X, Zhou Y, Feng Y, Yang Y, Wang X. Intrathecally administered Apelin-13 alleviated complete Freund's adjuvantinduced inflammatory pain in Mice. Front Pharmacol. 2020;11:1335. doi:10.3389/fphar.2020.01335

26. Ni H, Xu M, Xie K, et al. Liquiritin alleviates pain through inhibiting CXCL1/CXCR2 signaling pathway in bone cancer pain rat. Front Pharmacol. 2020;11:436. doi:10.3389/fphar.2020.00436

27. Kapralov A, Yang Q, Dar H, et al. Redox lipid reprogramming commands susceptibility of macrophages and microglia to ferroptotic death. Nat Chem Biol. 2020;16(3):278-290. doi:10.1038/s41589-0190462-8

28. Wang W, Naveed M, Baig M, Abbas M, Xiaohui Z. Experimental rodent models of chronic prostatitis and evaluation criteria. Biomed Pharmacother. 2018;108:1894-1901. doi:10.1016/j.biopha.20 18.10.010

29. Wang $\mathrm{X}$, Zhong $\mathrm{S}, \mathrm{Xu} \mathrm{T}$, et al. Histopathological classification criteria of rat model of chronic prostatitis/chronic pelvic pain syndrome. Int Urol Nephrol. 2015;47(2):307-316. doi:10.1007/ s11255-014-0868-x

30. Liu Y, Wazir J, Tang M, et al. Experimental autoimmune prostatitis: different antigens induction and antigen-specific therapy. Int Urol Nephrol. 2021;53(4):607-618. doi:10.1007/s11255-020-02703-8

31. Kawabata A. Prostaglandin E2 and pain-an update. Biol Pharm Bull. 2011;34(8):1170-1173. doi:10.1248/bpb.34.1170

32. Kirby R, Carson C, Dasgupta P. Daily phosphodiesterase type 5 inhibitor therapy: a new treatment option for prostatitis/prostatodynia? BJU Int. 2014;113(5):694-695. doi:10.1111/bju.12681

33. Wong L, Done J, Schaeffer A, Thumbikat P. Experimental autoimmune prostatitis induces microglial activation in the spinal cord. Prostate. 2015;75(1):50-59. doi:10.1002/pros.22891
34. Qiao L, Tiwari N. Spinal neuron-glia-immune interaction in cross-organ sensitization. Am J Physiol Gastrointest Liver Physiol. 2020;319:G748-G760. doi:10.1152/ajpgi.00323.2020

35. Turkington D, Grant J, Ferrier I, Rao N, Linsley K, Young A. A randomized controlled trial of fluvoxamine in prostatodynia, a male somatoform pain disorder. J Clin Psychiatry. 2002;63 (9):778-781. doi:10.4088/jcp.v63n0905

36. Du H, Chen X, Zhang L, et al. Microglial activation and neurobiological alterations in experimental autoimmune prostatitis-induced depressive-like behavior in mice. Neuropsychiatr Dis Treat. 2019;15:2231-2245. doi:10.2147/ndt.s211288

37. Wang Z, Chen M, Wang B, et al. Electroacupuncture alleviates osteoarthritis by suppressing NLRP3 inflammasome activation in guinea pigs. eCAM. 2020;2020:5476064. doi:10.1155/2020/5476064

38. Yu M, Wei R, Zhang T, et al. Electroacupuncture relieves pain and attenuates inflammation progression through inducing IL-10 production in CFA-induced mice. Inflammation. 2020;43(4):1233-1245. doi:10.1007/s10753-020-01203-2

39. Zhao J, Li H, Shi C, Yang T, Xu B. Electroacupuncture inhibits the activity of astrocytes in spinal cord in rats with visceral hypersensitivity by inhibiting $\mathrm{P} 2 \mathrm{Y}$ receptor-mediated MAPK/ERK signaling pathway. eCAM. 2020;2020:4956179. doi:10.1155/2020/4956179

40. Michael B, Bricio-Moreno L, Sorensen E, et al. Astrocyte- and neuron-derived CXCL1 drives neutrophil transmigration and blood-brain barrier permeability in viral encephalitis. Cell Rep. 2020;32(11):108150. doi:10.1016/j.celrep.2020.108150

41. Zheng Y, Zhou Y, Wu Q, et al. Effect of electroacupuncture on the expression of $\mathrm{P} 2 \times 4$, GABAA $\gamma 2$ and long-term potentiation in spinal cord of rats with neuropathic pain. Brain Res Bull. 2020;162:1-10. doi:10.1016/j.brainresbull.2020.04.020
Journal of Pain Research

\section{Publish your work in this journal}

The Journal of Pain Research is an international, peer reviewed, open access, online journal that welcomes laboratory and clinical findings in the fields of pain research and the prevention and management of pain. Original research, reviews, symposium reports, hypothesis formation and commentaries are all considered for publication. The manuscript management system is completely online and includes a very quick and fair peer-review system, which is all easy to use. Visit http:// www.dovepress.com/testimonials.php to read real quotes from published authors. 